\title{
光氧化还原促进的新型自由基硼化反应合成有机嗍化合物
}

\author{
许荷欢江智勇* \\ (河南师范大学化学化工学院 河南新乡 453007)
}

\section{Photoredox-Catalysed New Radical Borylation Pathways for Organoboron Synthesis}

\author{
$\mathrm{Xu}$, Hehuan Jiang, Zhiyong*
}

(School of Chemistry and Chemical Engineering, Henan Normal University, Xinxiang, Henan 453007)

有机嗍化合物被广泛应用于化学合成、材料科学和 药物化学等领域 ${ }^{[1]}$. 虽然目前已经存在大量的硼试剂和 合成方法来合成有机硼化合物 ${ }^{[2]}$, 但是, 探索新的反应 机理、发展广泛实用的硼化方法、获得多官能团化的有 机硼化合物仍然是合成化学的主要目标.

近年来, 嗍自由基参与的硼化反应研究受到了广泛 关注 ${ }^{[3]}$, 特别是氮杂环卡宾一硼自由基 $\left(\mathrm{NHC}-\mathrm{BH}_{2} \bullet\right.$ ) 作 为一种具有特殊活性的反应物种参与各种重要的合成 和催化反应. Curran ${ }^{[4]} 、$ Taniguchi $^{[5]}$ 、Walton ${ }^{[6]}$ 和 Wang ${ }^{[7]}$ 等课题组发展了一系列关于烯烃和炔烃的自由基硼化 反应, 用于合成多种有机硼化合物(Schme 1, a). 在这些
转化过程中, $\mathrm{NHC}-\mathrm{BH}_{2} \bullet$ 表现出传统离子反应和过渡金 属催化反应没有化学反应特性. 但是这些方法仍然具有 一定的局限性: 一方面, $\mathrm{NHC}-\mathrm{BH}_{2} \bullet$ 反应体系仅限于特 定结构的烯烃和炔烃; 另一方面, $\mathrm{NHC}-\mathrm{BH}_{2} \bullet$ 的产生主 要依赖偶氮类或过氧类等自由基引发剂的热裂解撜氢, 且该过程一般伴随着较高的引发温度. 为了进一步探究 氮杂卡宾-嗍自由基的合成应用, 迫切需要发展新的氮 杂卡宾-嗍自由基产生方式及反应类型.

随着光致氧化还原催化在单电子转移化学中的应用, $\mathrm{Zhu}$ 和 $\mathrm{Xie}^{[8]}$ 及 $\mathrm{Wu}^{[9]}$ 等利用光催化剂和硫醇协同催化, 将 $\mathrm{NHC}-\mathrm{BH}_{3}$ 转化为 $\mathrm{NHC}-\mathrm{BH}_{2} \cdot$, 实现了亚胺的反式嗍氢

(a) Previous work

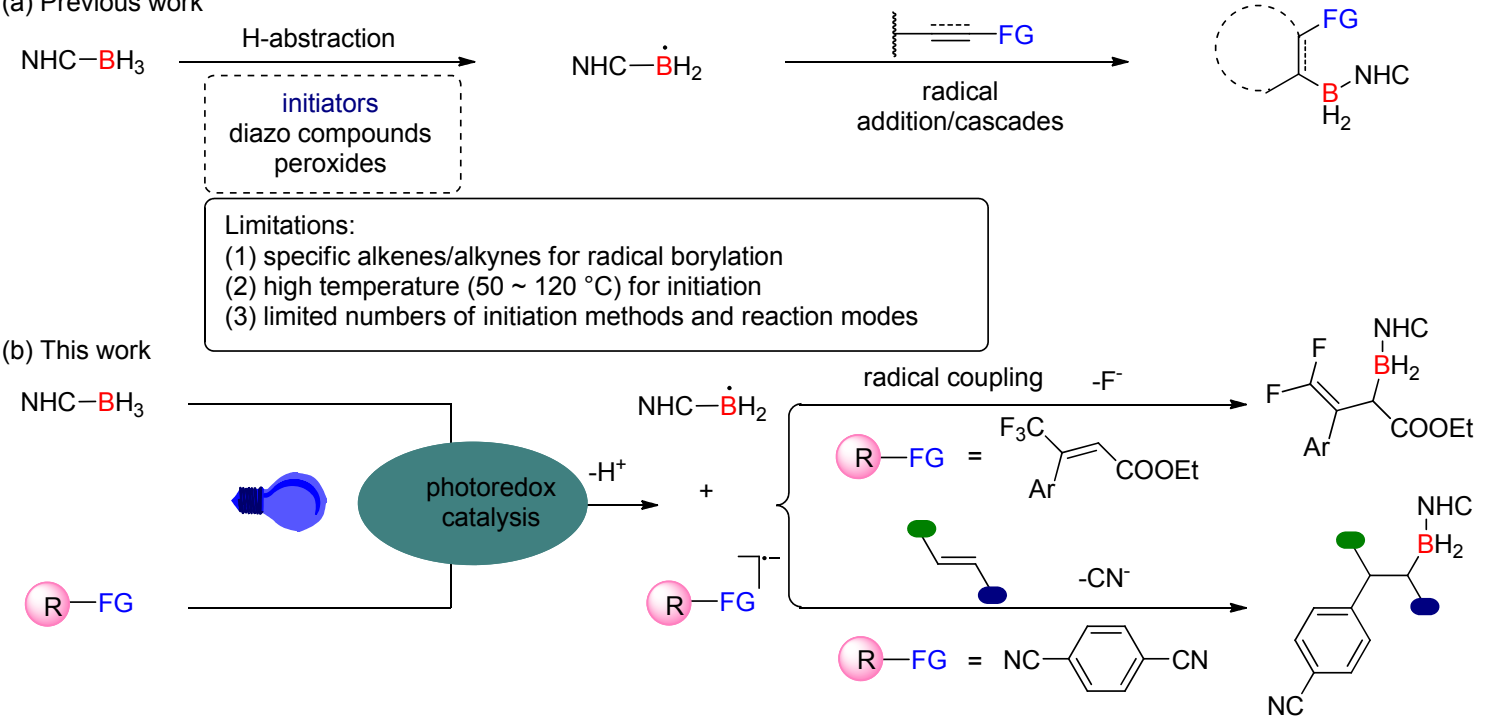

图式 $1 \mathrm{NHC}-\mathrm{BH}_{2} \cdot$ 合成有机硼化合物的反应

Scheme 1 Reactions of NHC-boryl radicals for the synthesis of organoboron molecules

* Corresponding author. E-mail: jiangzhiyong@htu.edu.cn. Published online September 2, 2020. 
化和多氟代芳烃、偕二氟烯烃及三氟甲基取代的端位烯 烃的去氟硼化反应. 但是此类方法仍然是通过篗氢的方 式产生氮杂卡宾-嗍自由基. 因此, 发展硼自由基新的产 生方式及设计新的硼化反应路径具有重要的意义.

汪义丰课题组 ${ }^{[10]}$ 一直致力于发展新型的自由基硓 化方法来制备有机喼化合物. 通过对 $\mathrm{NHC}-\mathrm{BH}_{3}$ 氧化电 势的研究, 利用可见光氧化策略从 $\mathrm{NHC}-\mathrm{BH}_{3}$ 直接产生 $\mathrm{NHC}-\mathrm{BH}_{2} \cdot$, 通过选择性的自由基偶联和自由基加成 实现三氟甲基取代的 $\alpha, \beta$-不饱和羰基化合物的去氟硼化 和芳基烯烃的芳硼化反应(Schme 1, b).

该反应条件温和, 不需要加入任何自由基引发剂和 氢原子转移试剂; 底物范围广, 官能团兼容性强; 化学
和区域选择性好，还可以实现生物活性分子和天然产物 的嗍化; 反应可以放大到克级, 得到的有机嗍化合物可 以进行多种衍生化，合成多种有用的化合物.

基于以上实验结果，作者提出了可能的反应机理. 首先, 激发态的光催化剂 $\left(\mathrm{Ir}^{\mathrm{III}}\right)$ 与 $\mathbf{2 a}$ 或者 $\mathbf{4 a}$ 发生氧化淬 灭生成 $\mathrm{Ir}^{\mathrm{IV}}$ 和自由基阴离子(III 或者 $\mathbf{V}$ ), 接着, $\mathrm{Ir}^{\mathrm{IV}}$ 氧化 $\mathrm{NHC}-\mathrm{BH}_{3}$, 快速脱质子生成 $\mathrm{NHC}-\mathrm{BH}_{2} \bullet(\mathbf{I I})$, 最后, $\mathrm{NHC}-\mathrm{BH}_{2}$-进行选择性的自由基反应得到相应的产物 $3 \mathbf{a}$ 和 $6 \mathbf{a}$ (Scheme 2).

在该工作发表的同一时期, Yang ${ }^{[11]}$ 和 Curran ${ }^{[12]}$ 等也 报道了类似的可见光促进的氧化产生 $\mathrm{NHC}-\mathrm{BH}_{2} \bullet$ 的工 作.

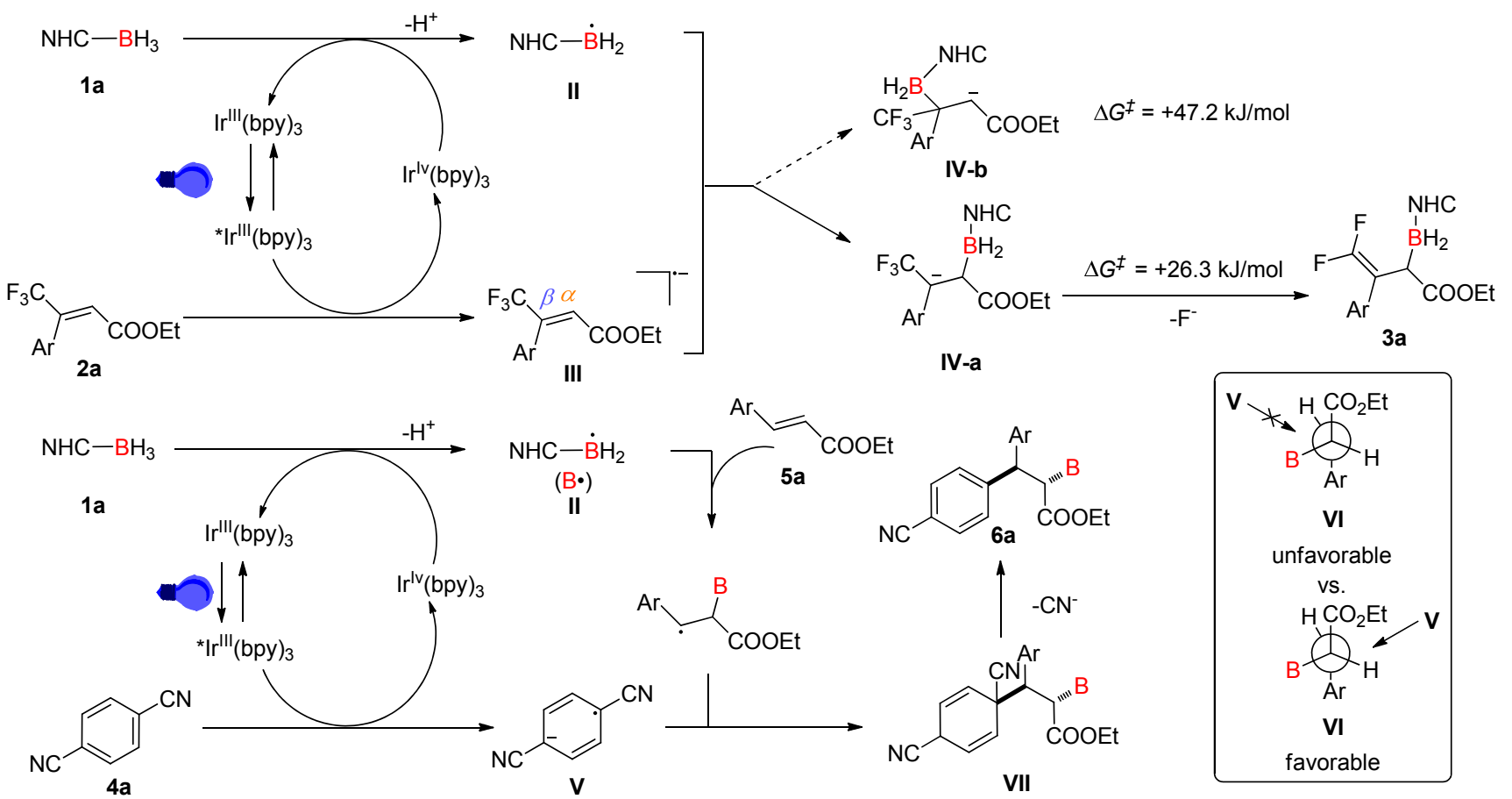

图式 2 反应机理

Scheme 2 Proposed mechanisms

\section{References}

[1] Hall, D. G. Boronic Acids: Preparation and Applications in Organic Synthesis Medicine and Materials, 2nd ed., Wiley-VCH, Weinheim, 2011.

[2] Collins, B. S. L.; Wilson, C. M.; Myers, E. L.; Aggarwal, V. K. Angew. Chem., Int. Ed. 2017, 56, 11700.

[3] (a) Jin, J.-K.; Xia, H.-M.; Zhang, F.-L.; Wang, Y.-F. Chin. J. Org. Chem. 2020, 40, 2185 (in Chinese).

(靳继康, 夏慧敏, 张凤莲, 汪义丰, 有机化学, 2020, 40, 2185.)

(b) Taniguchi, T. Eur. J. Org. Chem. 2019, 2019, 6308.

(c) Xu, A.-Q.; Zhang, F.-L.; Ye, T.; Yu, Z.-X.; Wang, Y.-F. CCS Chem. 2019, 1, 504.

[4] Dai, W.; Geib, S. J.; Curran, D. P. J. Am. Chem. Soc. 2019, 141, 12355.

[5] Shimoi, M.; Maeda, K.; Geib, S. J.; Curran, D. P.; Taniguchi, T.
Angew. Chem., Int. Ed. 2019, 58, 6357.

[6] Dai, W.; McFadden, T. R.; Curran, D. P.; Früchtl, H. A.; Walton, J. C. J. Am. Chem. Soc. 2018, 140, 15868.

[7] Ren, S.-C.; Zhang, F.-L.; Qi, J.; Huang, Y.-S.; Xu, A.-Q.; Yan, H.-Y.; Wang, Y.-F. J. Am. Chem. Soc. 2017, 139, 6050.

[8] Zhou, N.; Yuan, X.-A.; Zhao, Y.; Xie, J.; Zhu, C. Angew. Chem., Int. Ed. 2018, 57, 3990.

[9] Xu, W.; Jiang, H.; Leng, J.; Ong, H.-W.; Wu, J. Angew. Chem., Int. Ed. 2020, 59, 4009.

[10] Qi, J.; Zhang, F.-L.; Jin, J.-K.; Zhao, Q.; Li, B.; Liu, L.-X.; Wang, Y.-F. Angew. Chem., Int. Ed. 2020, 59, 12876.

[11] Xia, P. J.; Song, D.; Ye, Z. P.; Hu, Y. Z.; Xiao, J. A.; Xiang, H. Y.; Chen, X. Q.; Yang, H. Angew. Chem., Int. Ed. 2020, 59, 6706.

[12] Dai, W.; Geib, S. J.; Curran, D. P. J. Am. Chem. Soc. 2020, 142, 6261. 